\title{
Contrôle des cellules de Langerhans par le GM-CSF
}

La cellule de Langerhans est une cellule dendritique qui initie la réponse immunitaire spécifique de l'antigène. Elle capte l'antigène au niveau des épithéliums, puis migre dans les ganglions lymphatiques, où elle présente les déterminants antigéniques aux cellules $T$. Le granulocyte/macrophage colony-stimulating factor (GM-CSF) induit la prolifération et la différenciation des précurseurs des cellules de Langerhans, ainsi que l'activation des cellules de Langerhans in vitro. Il induit également l'accumulation des cellules de Langerhans in vivo. Cette cytokine joue donc un rôle majeur dans la régulation de la phase initiale de la réponse immune.

Jean-François Émile Michel Peuchmaur Nicole Brousse

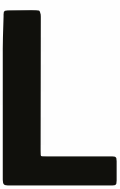

es cellules de Langerhans sont des cellules dendritiques dérivées de la moelle osseuse. Elles sont caractérisées par la présence de micro-organites cytoplasmiques appelés granules de Birbeck. En outre, elles expriment à leur surface les molécules de classe II du complexe majeur d'histocompatibilité (CMH) et l'antigène CDla [1]. Les cellules de Langerhans sont localisées dans la région supra-basale des épithéliums malpighiens de la peau et des muqueuses, dans l'urothélium et l'épithélium bronchique. Les cellules de Langerhans initient la réponse immune spécifique de l'antigène [2]. En effet, elles sont capables de capter les antigènes et de les internaliser. L'antigène est ensuite apprêté, c'est-à-dire partiellement dégradé en petits peptides qui sont associés aux molécules de classe II du CMH. Pendant ce temps, les cellules de Langerhans ont migré dans la zone paracorticale $\mathrm{T}$ des ganglions lymphatiques de drainage; elles prennent alors le nom de cellules dendritiques interdigitées. Les déterminants antigéniques sont alors exprimés à la surface cellulaire, associés aux molécules du $\mathrm{CMH}$. Les cellules interdigitées peuvent exprimer l'antigène $\mathrm{CDl}$, mais ne contiennent plus de granules de Birbeck. Ces cellules présentent les déterminants antigéniques aux lymphocytes T. Les mécanismes de capture et de présentation de l'antigène aux cellules $T$ sont maintenant assez bien compris. En revanche, les signaux moléculaires d'activation des cellules de Langerhans, et notamment ceux qui induisent la capture de l'antigène et la migration des cellules de Langerhans vers les ganglions de drainage, sont encore mal connus. Une telle connaissance est capitale, car elle permettrait, à 


\section{RÉFÉRENCES}

1. Thivolet J, Schmitt D. The Langerhans cell. Paris: Inserm/John Libbey Eurotext, 1988.

2. Schmitt D, Dezutter-Dambuyant C, Staquet MJ, Thivolet J. La cellule de Langerhans. médecine/sciences 1989 ; 5 : 103-11.

3. Lieschke GJ, Burgess AW'. Granulocyte colony-stimulating factor and granulocyte/macrophage colony-stimulating factor (first of two parts). N Engl / Med 1992 ; 327: 28-35.

4. Gough NM, Nicola NA. Granu locyte/macrophage colony stimulating factor. Immunol Ser $1990 ; 49$ : 111-53.

5. Caux C, Dezutter-Dambuyant C, Schmit D, Banchereau J. GM-CSF and TNF $\alpha$ cooperate in the generation of dendritic Langerhans cells. Nature 1992; 360 : 258-61.

6. Inaba $\mathrm{K}$, Inaba $\mathrm{M}$, Degushi $\mathrm{M}$, et al Granulocytes, macrophages, and dendritic cells arise from major histocompatibility complex class II-negative progenitor in mouse bone marrow. Proc Natl Acad Sci USA 1993; 90 : 3038-42.

7. Heufler C, Koch F, Schuler G. Granu locyte/macrophage colony-stimulating factor and interleukin 1 mediate the maturation of murine epidermal Langerhans cells into potent immunostimulatory dendritic cells. J Exp Med 1988; 167: 700-5

8. Witmer-Pack MD, Olivier W, Valinsky J, Schuler G, Steinman RM. Granulocyte/macrophage colony-stimulating factor is essential for the viability and function of cultured murine epidermal Langerhans cells. J Exp Med 1987; 166 : 1484-98.

9. Symington FW, Brady W', Linsley PS. Expression and function of $\mathrm{B} 7$ on human epidermal Langerhans cells. I Immuno $1993 ; 150$ : 1286-95.

10. Linsley PS, Ledbetter JA. The role of the C.D28 receptor during $T$ cell responses to antigen. Annu Rev Immunol 1993 I l : 191-212.

11. Morrissey PJ, Bressler L, Park LS, Alpert A, Gillis S. Granulocyte-macrophage colony-stimulating factor augments the primary response by enhancing the function of antigen-presenting cells. J Immunol 1987 139 : 1113-9.

12. Tao MH, Levy R. Idiotype/granulocytemacrophage colony-stimulating factor fusion protein as a vaccine for B-cell terme, d'activer, ou au contraire d'inhiber la réponse immune spécifique de l'antigène.

Le granulocyte/macrophage colonystimulating factor (GM-CSF) stimule la prolifération et la différenciation des progéniteurs hématopoiétiques, et induit la formation de colonies de polynucléaires neutrophiles, éosinophiles et de macrophages [3]. Cette protéine est produite par plusieurs types cellulaires: lymphocytes T activés, macrophages, cellules endothéliales, fibroblastes et kératinocytes [4]. L.es données concernant les effets du GM-CSF sur les cellules de Langerhans, plus récentes, suggèrent que cette cytokine joue un rôle central dans l'activation des cellules de Langerhans.

\section{Le GM-CSF induit la différenciation et l'activation des cellules de Langerhans in vitro}

Des chercheurs lyonnais ont cultivé des progéniteurs hématopoiétiques humains en présence de GM-CSF et de TNF $\alpha$ [5]. Après douze jours de culture, ils ont observé la présence d'une proportion importante de cellules dendritiques. Ces cellules ont les caractéristiques morphologiques, ultrastructurales, et immunocytochimiques des cellules de Langerhans. En outre, elles ont des capacités de présentation de l'antigène comparables à celles des cellules de Langerhans activées. Il reste à préciser le site de différenciation des cellules de Langerhans: soit celle-ci se déroule en grande partie dans la moelle ; soit, au contraire, la sécrétion de GM-CSF et de TNF $\alpha$ au niveau des sites inflammatoires induit la différenciation sur place de progéniteurs circulants. D'autres auteurs ont montré que le GM-CSF seul pouvait induire la différenciation de cellules dendritiques à partir de précurseurs hématopoïétiques murins [6].

Lorsque les cellules de Langerhans sont extraites de l'épiderme humain ou murin, la présence de GM-CSF est nécessaire à leur maintien en culture in vitro [7]. En outre, la culture de cellules de Langerhans en présence de GM-CSF augmente leur capacité de présentation de l'antigène. Lorsque des cellules de Ian- gerhans murines sont cultivées trois jours en présence de GM-CSF, les réponses en réactions lymphocytaires mixtes sont augmentées de dix à vingt fois [8]. Le GM-CSF augmente l'expression des antigènes de classe II du $\mathrm{CMH}$ dans les douze premières heures de culture, alors que la maturation fonctionnelle n'apparaît que plus tardivement [7]. L'activation des capacités de présentation ne dépend donc pas que de l'hyperexpression des antigènes de classe II du $\mathrm{CMH}$, et fait intervenir au moins un autre facteur. Ce co-facteur de stimulation pourrait être la molécule B7/BB1. En effet, cette molécule favorise l'activation des cellules $\mathrm{T}$ en se liant à la molécule CD28, et est détectable à la surface des cellules de Langerhans activées [9]. Ia molécule $\mathrm{B} 7 / \mathrm{BBl}$ se lie également à l'antigène CTLA-4 qui est exprimé par les lymphocytes T activés [10].

\section{Le GM-CSF favorise la réponse immunitaire humorale}

L'injection intraveineuse de GM-CSF augmente la réponse anticorps de souris contre des globules rouges de mouton [11]. L'injection intrapéritonéale d'une immunoglobuline monoclonale autologue à des souris n'est pas immunogène. En revanche, l'adjonction d'un adjuvant permet d'induire une réponse antiidiotypique. L'injection d'une molécule hybride idiotype/GM-CSF permet d'induire une réponse humorale primaire et secondaire d'intensité au moins égale à celle obtenue avec un adjuvant classique [12]. Sachant que le GM-CSF active in vitro les capacités de présentation antigénique, son effet adjuvant in vivo pourrait être lié à l'activation de cellules dendritiques ou de cellules de Iangerhans.

\section{Le GM-CSF induit une accumulation locale de cellules de Langerhans}

L'intensité de la réponse immune dépend probablement de la densité en cellules de Langerhans au site de pénétration de l'antigène. En effet, l'irradiation de l'épiderme humain par des rayons ultraviolets réduit la 

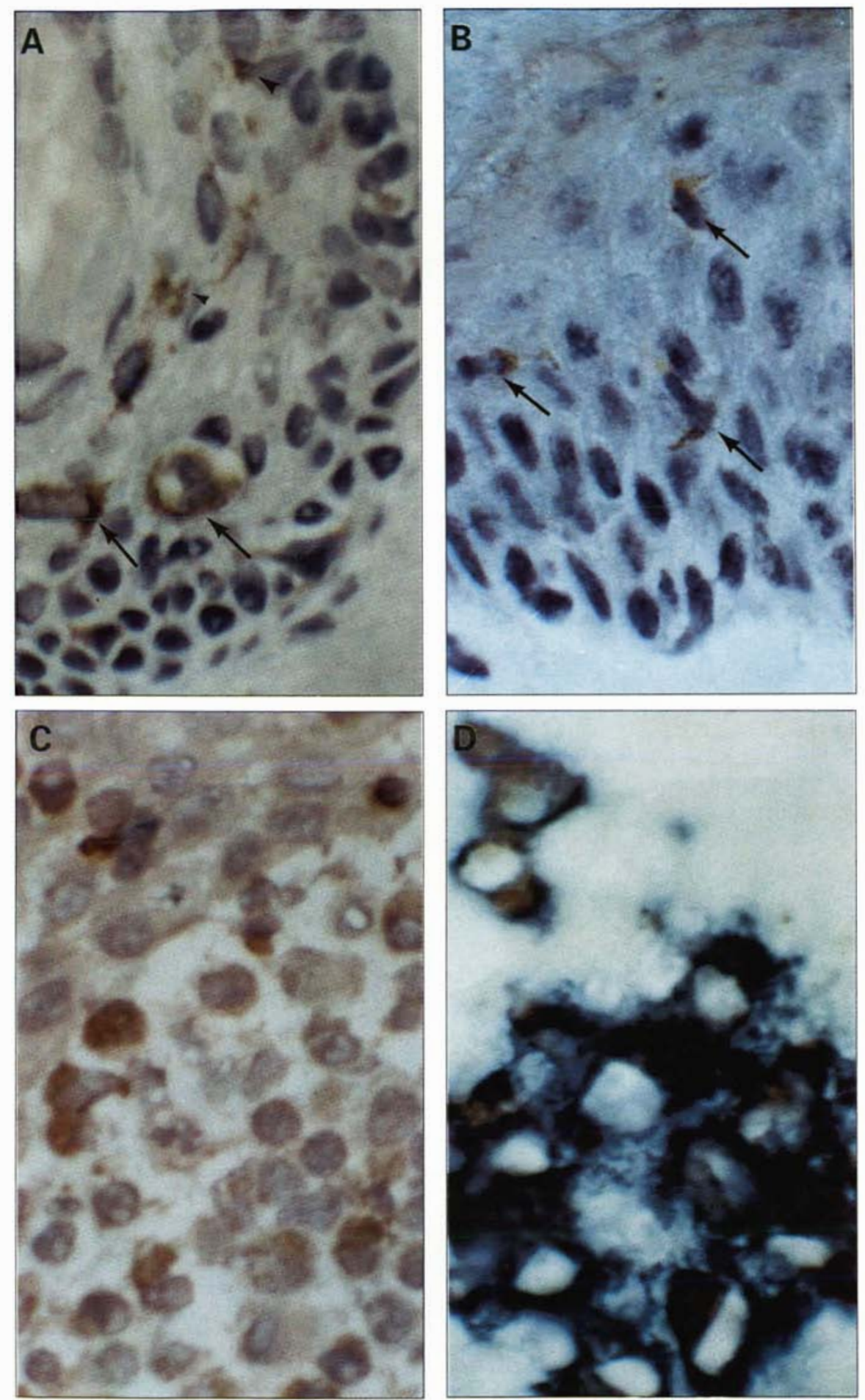

Figure 1. Détection par immunohistochimie du GM-CSF dans le cytoplasme des cellules de Langerhans. A. Follicules pileux d'un patient atteint de pelade: les cellules de Langerhans (flèche) et leurs expansions dendritiques (têtes de flèches) sont marquées en brun. B. Dans la peau normale, quelques cellules de Langerhans (flèches) contiennent du GM-CSF. C. Prélèvement cutané d'un patient atteint d'histiocytose langerhansienne: les cellules de Langerhans tumorales infiltrent la partie superficielle du derme; leur cytoplasme est marqué par l'anticorps anti-GM-CSF. D. Prélèvement cutané d'un patient atteint d'histiocytose langerhansienne: double marquage avec l'anticorps anti-CD1a (marquage membranaire en bleu), et avec un anticorps anti-GM-CSF (marquage cytoplasmique en brun). Les cellules contenant du GM-CSF sont des cellules de Langerhans tumorales.

$\mathrm{m} / \mathrm{s} n^{\circ} 2$ vol. 10, feurie 94 densité en cellules de Langerhans, et diminue parallèlement l'intensité de la réaction au dinitrochlorobenzène [13]. Des extraits d'épiderme murin traité localement par des agents inflammatoires ont une activité GM-CSF détectable [14].

Chez les patients atteints de pelade, le derme est le siège d'un infiltrat inflammatoire, et on observe une accumulation de cellules de Langerhans, notamment dans les follicules pileux. Nous avons montré que, chez ces patients, beaucoup de ces cellules de Langerhans contiennent du GM-CSF (figure 1A). Le GM-CSF est détecté dans le cytoplasme avec deux anticorps monoclonaux antiGM-CSF reconnaissant deux épitopes différents de cette cytokine. Dans l'épiderme de patients témoins, nous n'avons détecté de GM-CSF que dans de rares cellules de Langerhans (figure 1B).

Enfin, l'administration intradermique de GM-CSF à des patients atteints de lèpre induit une accumulation locale de cellules de Langerhans [15]. Le nombre maximum de cellules de Langerhans est observé au quatrième jour après l'injection. Cette accumulation peut être due, soit à la prolifération des cellules de Langerhans résidentes, soit à la différenciation locale de progéniteurs, soit au recrutement de cellules de Langerhans circulantes. L'ensemble de ces résultats suggère que la production locale de GM-CSF pourrait être le mécanisme physiologique de recrutement des cellules de Langerhans.

\section{Rôle du GM-CSF dans la pathogénie de I'histiocytose langerhansienne}

Nous avons recherché la présence de GM-CSF chez des patients atteints d'histiocytose langerhansienne. Cette maladie, qui touche généralement l'enfant, est caractérisée par la présence de granulomes contenant de nombreuses cellules de Langerhans. Elle peut être localisée, touchant fréquemment la peau ou l'os, ou être diffuse à plusieurs organes. Nous avons pratiqué un marquage immunohistochimique sur des coupes congelées d'histiocytose langerhansienne avec deux anticorps monoclonaux 


\section{RÉFÉRENCES}

13. Cooper KD, Oberhelman L, Hamilton $\mathrm{TA}$, et al. UV exposure reduces immunization rates and promotes tolerance to epicutaneous antigens in humans : relationship to dose, CDla-DR+ epidermal macrophage induction, and Langerhans cell depletion. Proc Natl Acad Sci USA 1992; 89 : 8497-501.

14. Koury MJ, Balmain A, Pragnell IB Induction of granulocyte/macrophage colony-stimulating activity in mouse skin by inflammatory agents and tumor promoters. ЕМВО / 1983 ; 2 : 1877-82

15. Kaplan G, Walsh G, Guido LS, et al. Novel responses of human skin to intradermal recombinant granulocyte/macrophage colony-stimulating factor: Langerhans cell recruitment, keratinocyte growth, and enhanced wound healing. / Exp Med 1992 175: 1717-28

16. Émile JF, Peuchmaur M, Fraitag S, Bodemer C, Brousse N. Immunohistochemical detection of GM-CSF in human Langerhans' cells. Histopathology 1993; 23 $327-32$.

17. Lang RA, Metcalf D, Cuthbertson RA, et al. Transgenic mice expressing a hematopoietic growth factor gene (GM-C.SF) develop accumulations of macrophages blindness, and a fatal syndrome of tissue damage. Cell 1987; 51 : 675-86.

18. Metcalf D, Moore JG. Divergent disease patterns in granulocyte/macrophage colony-stimulating factor transgenic mice associated with different transgene insertion sites. Proc Natl Acad Sci USA 1988; 85 $7767-71$

19. Wilson PA, Ayscue LH, Jones GR, et al. Bone marrow histiocytic proliferation in association with colony-stimulating factor therapy. Am J Clin Pathol 1993; 99 : 311-3.

20. Hammar S, Bockus D, Remington F, Bartha $M$. The widespread distribution of Langerhans cells in pathologic tissues : an ultrastructural and immunohistochemical study. Hum Pathol 1986; 17: 894-905.

21. Tazi A, Bouchonnet F, Grandsaigne M, Boumsell L, Hance AJ, Soler P. Evidence that granulocyte/macrophage colonystimulating factor regulates the distribution and differentiation state of dendritic cells/Langerhans cells in human lung and lung cancers. J Clin Invest 1993; 91 : 566-76.

22. Dranoff G, Jaffee E, Lazenby A, et al. Vaccination with irradiated tumor cells engineered to secrete murine granulocyte/macrophage colony-stimulating factor stimulates potent, specific, and longlasting anti-tumor immunity. Proc Natl Acad Sci USA 1993; 90): 3539-43.

23. Schreiber S, Kilgus O, Payer E, et al. Cytokine pattern of Langerhans cells isolated from murine epidermal cell cultures. I
anti-GM-CSF [16]. Ces deux anticorps reconnaissent des épitopes différents du GM-CSF, et n'ont pas de réaction croisée avec le M-CSF (monocyteCSF), le G-CSF (granulocyte-CSF), l'IL1 $\alpha$, l'IL1 $\beta$, l'IL2, l'IL3, le TNF $\alpha$, le TNF $\beta$ ni les interférons $\alpha, \beta$ et $\gamma$ en ELISA. Le marquage a été pratiqué par une technique d'immunohistochimie en trois couches. Les témoins, avec un premier anticorps de même isotype, et après omission du premier anticorps, étaient négatifs. Sur tous les prélèvement testés, cutanés ou osseux, du GM-CSF a été détecté dans les cellules de Langerhans tumorales (figure 1C). La nature des cellules marquées a été confirmée par un double marquage avec un anticorps anti-CDla (figure 1D). Le GM-CSF n'a pas été détecté dans d'autres types cellulaires. Nous avons également recherché la présence de GM-CSF dans le sérum d'enfants atteints d'histiocytose langerhansienne. Cette cytokine a une demi-vie très courte et n'est pas détectable chez les sujets sains. Nous avons détecté du GM-CSF dans le sérum d'enfants atteints de formes diffuses sévères d'histiocytose langerhansienne, mais non dans les formes localisées (J.F. Émile, E. Tartour, communication personnelle). Ce résultat préliminaire suggère que le GM-CSF pourrait être un marqueur de la masse tumorale. L'intérêt pronostique de ce marqueur sérique mérite d'être testé. L'ensemble de ces données permet de supposer que le GMCSF est un facteur de prolifération de l'histiocytose langerhansienne.

En faveur de cette hypothèse, il faut rappeler que les souris de deux lignées transgéniques pour le GMCSF meurent en quelques mois d'un infiltrat tissulaire diffus $[17,18]$. Cet infiltrat est constitué, comme celui de l'histiocytose langerhansienne humaine, de polynucléaires éosinophiles, de lymphocytes, et surtout de cellules mononucléées qui pourraient être des cellules de Langerhans. Toutefois, l'étude ultrastructurale et/ou l'immunomarquage de ces cellules n'ont jamais été publiés. Par ailleurs, un enfant traité par GM-CSF a présenté une infiltration diffuse de la moelle osseuse par des cellules mononucléées, qui n'ont pas non plus été phénotypées [19].

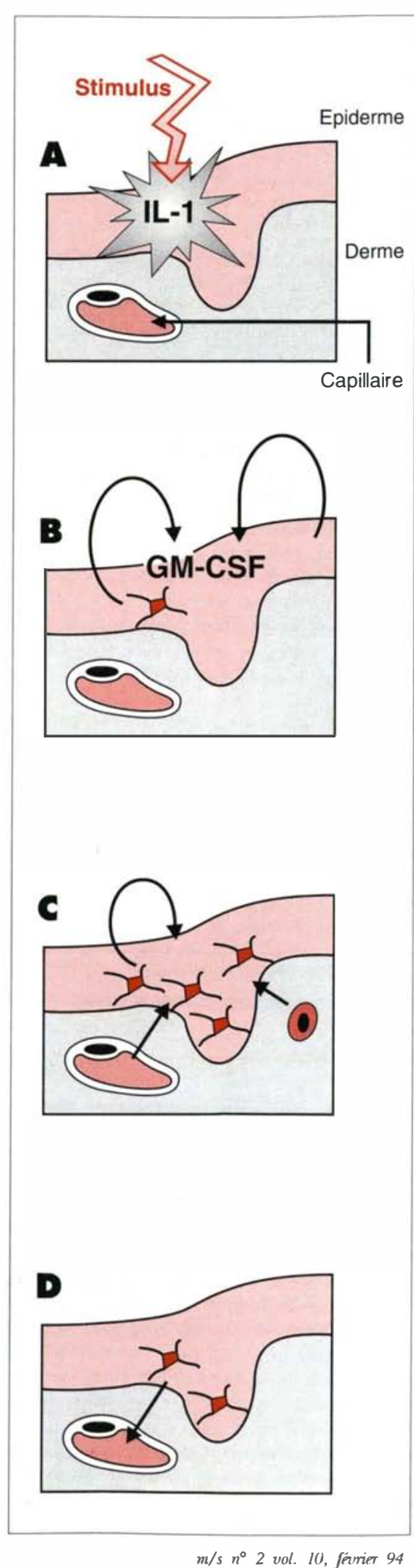




\section{Le GM-CSF induit une réponse immune antitumorale}

Les cellules de Langerhans sont détectables au sein de nombreuses tumeurs. Leur rôle dans l'initiation de la réponse antitumorale a été proposé [20]. Les cellules de Langerhans sont, en particulier, assez fréquemment rencontrées dans les carcinomes bronchiques. Leur densité est corrélée à la présence de GM-CSF, détecté au sein de la tumeur par immunohistochimie ou par hybridation in situ [21]. Enfin, des chercheurs ont transfecté une lignée de cellules tumorales avec diverses cytokines (IL2, IL3, IL4, IL6, interféron $\gamma$, TNF $\alpha$ et GM-CSF). Ces cellules ont ensuite été irradiées et injectées à des souris. Les cellules exprimant le GM-CSF, et seulement celles-ci, ont induit une immunité antitumorale durable et spécifique [22]

\section{Hypothèse physiologique}

L'action physiologique précise du GM-CSF sur les cellules de Langerhans est encore loin d'être déterminée. Toutefois, il est déjà possible, au vu des résultats récemment publiés, de formuler quelques hypothèses. La phase initiale de l'inflammation stimule la production locale de GM-CSF (figure 2). Cette stimulation est probablement relayée par l'ILl. Le rôle du TNF $\alpha$ à ce stade est encore discuté. Le GM-CSF pourrait être produit par les kératinocytes, comme cela a été montré chez la souris [23]. Il est également possible que, dans certaines maladies humaines telle l'histiocytose langerhansienne, le GM-CSF soit produit par les celllules de Langerhans elles-mêmes. La diffusion locale du GM-CSF induit ensuite l'accumulation des cellules de Langerhans. Celles-ci peuvent provenir, soit du recrutement de cellules de Langerhans circulantes, soit de la prolifération de cellules de Langerhans résidentes, soit enfin de la différenciation locale de progéniteurs intradermiques. Le GM-CSF s'accumule localement dans le cytoplasme des cellules de Langerhans. Les cellules de Langerhans activées vont alors capter l'antigène, migrer dans le ganglion de drainage, et présenter les déterminants antigéniques aux cellules $T$, initiant ainsi la réponse immunitaire spécifique de l'antigène.

Les données publiées au cours des six dernières années ont prouvé que le GM-CSF induit la prolifération et la différenciation des précurseurs des cellules de Langerhans, ainsi que l'activation des cellules de Langerhans in vitro. Il induit également l'accumulation des cellules de Langerhans in vivo. Cette cytokine joue donc un rôle majeur dans l'initiation de la réponse immune spécifique de l'antigène. Le GM-CSF pourrait, de ce fait, prendre une place importante dans les stratégies de vaccination anti-infectieuse ou antitumorale. Par ailleurs, des thérapeutiques anti-GM-CSF pourraient éventuellement être utiles au traitement de l'histiocytose langerhansienne

\section{Summary \\ Control of Langerhans cells by GM-CSF}

Langerhans cells are dendritic cells, which initiate the antigen specific immune response. They internalize and process the antigen. Then they migrate from the epithelium to the draining lymph node, where they present the antigen to the $\mathrm{T}$ cells. Granulocyte/macrophage colonystimulating factor (GM-CSF) induces differentiation, proliferation of Langerhans' cell precursors, and activation of Langerhans' cells in vitro. GM-CSF also induces accumulation of Langerhans' cells in vivo. Therefore, GM-CSF is one of the main immune response initiating factors.

\section{Remerciements}

Nous remercions S. Fraitag et E. Tartour pour leur participation à ce travail. Les deux anticorps anti-GM-CSF (10A8 et 14G12L1) et le kit de dosage sérique du GM-CSF ont été gracieusement fournis par le laboratoire Medgenix (Fleurus, Belgique). Ce travail a été financé en partie par un contrat normalisé d'étude pilote Inserm 93CN18.
Figure 2. Effets du GM-CSF sur les cellules de Langerhans: hypothèse physiologique. A. Initiation de l'inflammation: un stimulus externe induit la sécrétion de cytokines inflammatoires, particulièrement I'IL1. B. Production locale de GM-CSF: les cytokines inflammatoires induisent la sécrétion de GM-CSF par les kératinocytes, ou éventuellement par les cellules de Langerhans. C. Accumulation et activation des cellules de Langerhans: Ie GMCSF augmente la densité locale en cellules de Langerhans, par division des cellules de Langerhans résidentes, par différenciation de progéniteurs ou par recrutement des cellules de Langerhans circulantes. D. Migration des cellules de Langerhans: les cellules de Langerhans activées ont capté l'antigène et migrent vers le ganglion lymphatique de drainage.

$\mathrm{m} / \mathrm{s} n^{\circ} 2$ vol. 10, fevrier 94

\section{TIRÉS A PART}

J.F. Émile 